\title{
Behavioural activation written self-help to improve mood, wellbeing and quality of life in people with dementia supported by informal carers (PROMOTE): a study protocol for a single-arm feasibility study
}

\author{
Paul Farrand ${ }^{1 *}$, Joanne Woodford', David Llewellyn², Martin Anderson', Shanker Venkatasubramanian?',
} Obioha C. Ukoumunne ${ }^{3}$, Anna Adlam ${ }^{1}$ and Chris Dickens ${ }^{2}$

\begin{abstract}
Background: Increases in life expectancy have resulted in a global rise in dementia prevalence. Dementia is associated with poor wellbeing, low quality of life and increased incidence of mental health difficulties such as low mood or depression. However, currently, there is limited access to evidence-based psychological interventions for people with dementia experiencing low mood and poor wellbeing. Behavioural activation-based self-help, supported by informal carers and guided by mental health professionals, may represent an effective and acceptable solution.

Methods/design: The present study is a phase II (feasibility) single-arm trial informed by the Medical Research Council complex interventions research methods framework. Up to 50 dementia participant/informal carer dyads will be recruited from a variety of settings including primary care, dementia-specific health settings and community outreach. People living with dementia will receive behavioural activation-based self-help and be supported by their informal carer who has received training in the skills required to support the self-help approach. In turn, during the use of the intervention, the informal carer will be guided by mental health professionals to help them work through the materials and problem solve any difficulties. Consistent with the objectives of feasibility studies, outcomes relating to recruitment from different settings, employment of different recruitment methods, attrition, data collection procedures, clinical delivery and acceptability of the intervention will be examined. Clinical outcomes for people with dementia (symptoms of depression and quality of life) and informal carers (symptoms of depression and anxiety, carer burden and quality of life) will be measured pre-treatment and at 3 months post-treatment allocation.
\end{abstract}

Discussion: This study will examine the feasibility and acceptability of a novel behavioural activation-based self-help intervention designed to promote wellbeing and improve low mood in people living with dementia, alongside methodological and procedural uncertainties associated with research-related procedures. As determined by pre-specified progression criteria, if research procedures and the new intervention demonstrate feasibility and acceptability, results will then be used to inform the design of a pilot randomised controlled trial (RCT) to specifically examine remaining methodological uncertainties associated with recruitment into a randomised controlled design.

Trial registration: Current Controlled Trials ISRCTN42017211

Keywords: Dementia, Depression, Behavioural activation, Caregivers, Feasibility

\footnotetext{
* Correspondence: p.a.farrand@exeter.ac.uk

${ }^{1}$ Clinical Education Development and Research (CEDAR), Psychology: College of Life and Environmental Sciences, University of Exeter, Washington Singer Labs, Perry Road, Exeter EX4 4QG, UK

Full list of author information is available at the end of the article
} 


\section{Background}

Dementia is a global healthcare concern, with 115.4 million people worldwide expected to be living with dementia by 2050 [1]. Given there are no current cure or preventative medical interventions [2], dementia represents a significant challenge for health policy [3]. Current estimates indicate that on a global scale, prevalence stands at 35.6 million with 670,000 living with dementia in the United Kingdom (UK) [4]. The provision of long-term support to help people with dementia 'live well' is, therefore, a global health and social care priority [5-7].

Developing approaches to facilitate long-term support is especially important given quality of life, increased levels of mortality, increased health and social care costs [8] and poorer functional outcomes [9] commonly experienced by people with dementia [10]. Furthermore, between $30 \%[11,12]$ and $50 \%$ [13] of people with dementia also experience elevated symptoms of depression. However, despite depression being one of the most common mental health difficulties experienced by people living with dementia [14], access to evidence-based psychological therapies remains limited [15]. This treatment gap [16] exists despite growing evidence identifying cognitive behavioural therapy (CBT) as an effective intervention for treating depression in people with dementia [12], partly due to costs of delivery and a lack of trained therapists [17-19].

To provide a potential solution to address this treatment gap, CBT provided in a self-help format is being introduced into mental health services on a global scale [20-23]. CBT self-help is defined as CBT-specific therapeutic techniques being communicated in the form of bibliotherapy, online, audio or smartphone applications $[24,25]$ as opposed to delivery by a therapist [24, 26]. Some evidence suggests effectiveness increases when some form of face-to-face, telephone, or email guidance or support is also provided [26-28]. Within the Improving Access to Psychological Therapies (IAPT) Programme implemented across England [29], support is provided by a practitioner-based workforce (Psychological Wellbeing Practitioners (PWPs)) and introduced alongside face-toface 'high-intensity' evidence-based psychological therapies within a stepped care model of delivery [30]. Recent evidence also suggests support can be provided by nonprofessionals [31]. As such, a potential solution to increase access to psychological therapies for people with dementia may be for non-professionals, such as informal carers defined as those providing unpaid and untrained support within the community to a care recipient [32], to support therapy delivery. The provision of informal carer itself is associated with increased mental health difficulties such as anxiety and depression [33], restriction of social and recreational activities [34] and poor quality of life [35]. Conversely however, other evidence suggests that involving informal carers in the facilitation of psychological interventions for people living with dementia may also improve caregiver mood [36].

Behavioural activation (BA) is a psychological intervention featuring prominently within the IAPT programme [37] and is an evidence-based and cost-effective [38, 39] treatment for depression. BA aims to overcome depression through a structured and graded approach to reintroduce activity into people's lives to target behavioural avoidance [37] which is common in depression. Specifically, techniques used in BA help reintroduce people to sources of positive-reinforcement within their environment, whilst overcoming sources of negative reinforcement that maintain avoidance behaviours [40]. Several characteristics of the BA self-help protocol utilised within the IAPT programme [40] suggest it may also have potential utility for people with dementia. Not only is BA considered a straightforward approach making it easier for users with dementia and their carers to understand [41] but it may also complement the range of self-management techniques people with dementia and carer dyads already utilise [42]. Initial evidence has already highlighted the potential utility of BA as an effective intervention for treatment of depression in people with Alzheimer's disease, supported by a therapist and informal carer [36, 43]. However, these studies focused on the use of experienced geriatricians to deliver face-to-face therapy $[36,43]$, analogous to CBT 'high-intensity' support within a stepped care model of delivery [30]. Implementing this 'high-intensity' intervention delivered face-to-face by an experienced geriatrician workforce, who may already face immense demands on their time with other aspects of their role may, therefore, be prohibitive and unable to meet the potential demand for treatment [44].

A research programme informed by the Medical Research Council (MRC) complex interventions framework [45] has been undertaken to develop a written BA-based self-help intervention to target low mood and improve wellbeing in people with dementia, supported by their informal carers who themselves are guided in delivering the intervention by a practitioner-based PWP workforce. The current feasibility study builds upon MRC phase I development work [45] previously completed. Recognising the importance of involving people living with dementia and their carers actively in research [46], the phase I study involved semi-structured interviews with people with dementia to develop a new written BA-based self-help intervention to meet the needs of people with dementia. Additionally, two focus groups took place with carers about their role in supporting the person living with dementia work through the materials and problem solve any difficulties encountered under PWP guidance. This protocol represents MRC phase II (feasibility) research [45] to examine methodological, procedural and clinical uncertainties $[47,48]$. Should progression criteria for this feasibility study 
be met, results will inform the design and funding application for a further phase II (pilot) randomised controlled trial (RCT) that will examine methodological and procedural uncertainties specifically related to an RCT design. Subsequent progression of this research programme to a possible future definitive (phase III) RCT will be informed by results from the piloting phase.

\section{Study aims and objectives}

A single-arm feasibility phase II study [45] with an embedded qualitative component examining a number of feasibility questions pertaining to methodological, procedural and clinical uncertainties $[47,48]$ will be conducted.

The following outcomes will be examined following guidance concerning feasibility study objectives, as distinguished from objectives for a pilot RCT [47]:

1. The intensity of the recruitment procedure in terms of number of invitation packages sent by health professionals

2. Number of health professionals required to recruit into the study

3. The time taken (up to 6 months) to recruit the a priori determined sample size

4. Willingness of clinicians to recruit participants across multiple recruitment settings utilising different recruitment strategies

5. Participant response rates between different recruitment techniques

6. Eligibility proportions

7. Participant level barriers to recruitment.

8. Study resources required to implement study procedures

9. Feasibility and acceptability of data collection procedures to participants

10. Feasibility and acceptability of the intervention to participants and clinicians

11. Barriers to clinical delivery

12. Clinician training needs

13. Clinician adherence to the intervention protocol

As determined by pre-specified progression criteria, outcomes will be used to inform the design of a subsequent pilot RCT.

\section{Methods/design}

\section{Study design}

A feasibility phase II single-arm trial [45] with an embedded qualitative sub-study will be conducted. This protocol (version 1, 18/08/2015) is registered on Current Controlled Trials ISRCTN42017211 and follows Standard Protocol Items, Recommendations for Interventional Trial (SPIRIT) [49] guidelines for reporting interventional trials.

\section{Setting}

Four different settings-general practitioners (GPs), specialist dementia healthcare settings employing Primary Care Dementia Practitioners (PCDP), memory clinics and community outreach in the county of Cornwall (southwest England) - will be utilised to examine feasibility outcomes associated with recruitment. All treatment will be provided by PWPs within 'step 2' of an IAPT primary care mental health service adopting a stepped care model [30]. Recruitment setting locations have been selected on the basis of locality of study PWPs from the IAPT service to help increase the feasibility of intervention delivery. Further, recruitment setting types were selected on the basis of recruitment strategies utilised in other depression [50-52] and dementia studies $[53,54]$.

\section{Eligibility criteria}

People living with dementia

Participants are included if they:

1. Have a diagnosis of probable dementia recorded in medical records, with no restriction placed on dementia type given evidence suggesting mixed dementia is the most common dementia presentation [55]

2. Have mild-to-moderate dementia severity defined as scoring between 12 and 24 on the mini-mental state examination (MMSE) [56]

3. Have a score of 4 or more on the 12 -item Geriatric Depression Scale (GDS-12R) [57]

4. Residing at home

5. Able to provide informed consent or have an informal carer willing to provide consultee consent

6. Have sufficient proficiency in English to read and engage with the BA-based self-help material

7. Have an informal carer (defined as a partner, family member or friend) who has regular contact (at least weekly) with the person with dementia and willing to support the intervention.

Participants receiving antidepressant medication and/ or acetyl-cholinesterase inhibitors or memantine will also be able to participate if they have been receiving a stable dose for at least 1 month before recruitment. No upper or lower age restrictions are placed on people living with dementia.

Participants are excluded if they:

1. Have a co-morbid diagnosis of a severe and enduring mental health problem including psychosis, type I or II bipolar disorder and personality disorder recorded in medical records or self-reported to the study team 
2. Are currently receiving formal psychotherapy or another potentially active psychological treatment

3. Are acutely suicidal and/or have a history of persistent self-injury

4. Have self-report or medical record documented misuse of alcohol, prescription drugs or street drugs, so severe it interferes with the person with dementia's ability to perform normal activities in daily life and engagement with the intervention

Exclusions 1-3 are informed by guidance indicating that CBT self-help is not suitable for the specific population identified or that people with depression should only receive one psychological intervention at a time [58].

\section{Informal carers}

Participants are included if they are:

1. Aged 16 years or over.

2. Self-identified informal carer of a person with dementia with regular contact (at least weekly) with the person with dementia

3. Willing to support the intervention, including increasing contact to facilitate supporting the intervention if required

Participants are excluded if they:

1. Score over 20 on the Patient Health Questionnaire-9 (PHQ-9) indicating severe levels of depression [59]

2. Have a co-morbid diagnosis of a severe and enduring mental health problem including post-traumatic stress disorder (PTSD), psychosis, type I and II bipolar disorder or personality disorder as recorded in medical records or self-reported to the study team

3. Have self-report or medical record documented misuse of alcohol, prescription drugs or street drugs, so severe it interferes with the informal carer's ability to perform normal activities in daily life and engagement with the intervention

4. Have difficulties reading or following the written BA self-help material

5. Are acutely suicidal and/or have a history of persistent self-injury

The exclusion criteria for informal carers are based upon clinical factors that may impair and interfere with an informal carer's ability to support and engage in the intervention and indicate the carer themselves may require psychological support.

To be included within the study, both members of the dyad (person with dementia and informal carer) need to meet the inclusion criteria. In the case of a person with dementia being eligible for inclusion, but an informal carer ineligible, the researcher will work with the dyad to identify if another informal carer known to the dyad may be willing to participate in the study.

\section{Recruitment settings and procedure}

A multifaceted recruitment approach will be employed, building on techniques used to successfully recruit carers of people with dementia [54] across four specific recruitment settings (see Fig. 1).

\section{GP records}

GP search and mail-out will be adopted as used successfully in a number of depression trials [50-52]. GP electronic case records will be searched for people with a formal diagnosis of dementia, and a manual screen will be performed to check against the inclusion criteria. Reasons for exclusion will be anonymised and provided to the research team [60]. Participant invitation packages (study invitation letter, study summary sheet, reply slip and reasons for refusal of participation questionnaire) will be sent to people with dementia included in the screen. This package will also include a separate study invitation package to be passed onto an informal carer. People with dementia, or informal carers, will be able to contact the research team by returning the reply slip, telephoning or emailing the research team. Given that telephone reminders by clinicians have been reported to increase recruitment rates [61], non-responders will be provided with telephone follow-up calls by general practice staff. Where phone calls receive no answer, a maximum of four attempts to establish contact will be made over this time period. GPs can also directly refer suitable people with dementia. Study posters and brochures will also be displayed in practice receptions to further advertise the study.

\section{Primary care dementia practitioners}

PCDPs will perform a search and mail-out. Drawn from a variety of backgrounds in health care, PCDPs are health professionals specialising in dementia care providing support to people living with dementia and their families in the community. PCDPs will screen caseloads to check against the inclusion criteria. Reasons for exclusion will be anonymised and provided to the research team. Study invitation packages will be sent in a manner consistent with that used for GP record recruitment or handed to potential participants face-to-face. It is anticipated that PCDPs will complete their initial search and mail-out within 1 month of the study commencing recruitment with any patients added to their caseload over the 6-month recruitment period being invited faceto-face. PCDPs will provide non-responders with telephone reminders (maximum of four attempts) and can also directly refer suitable people with dementia during the course of the study. 


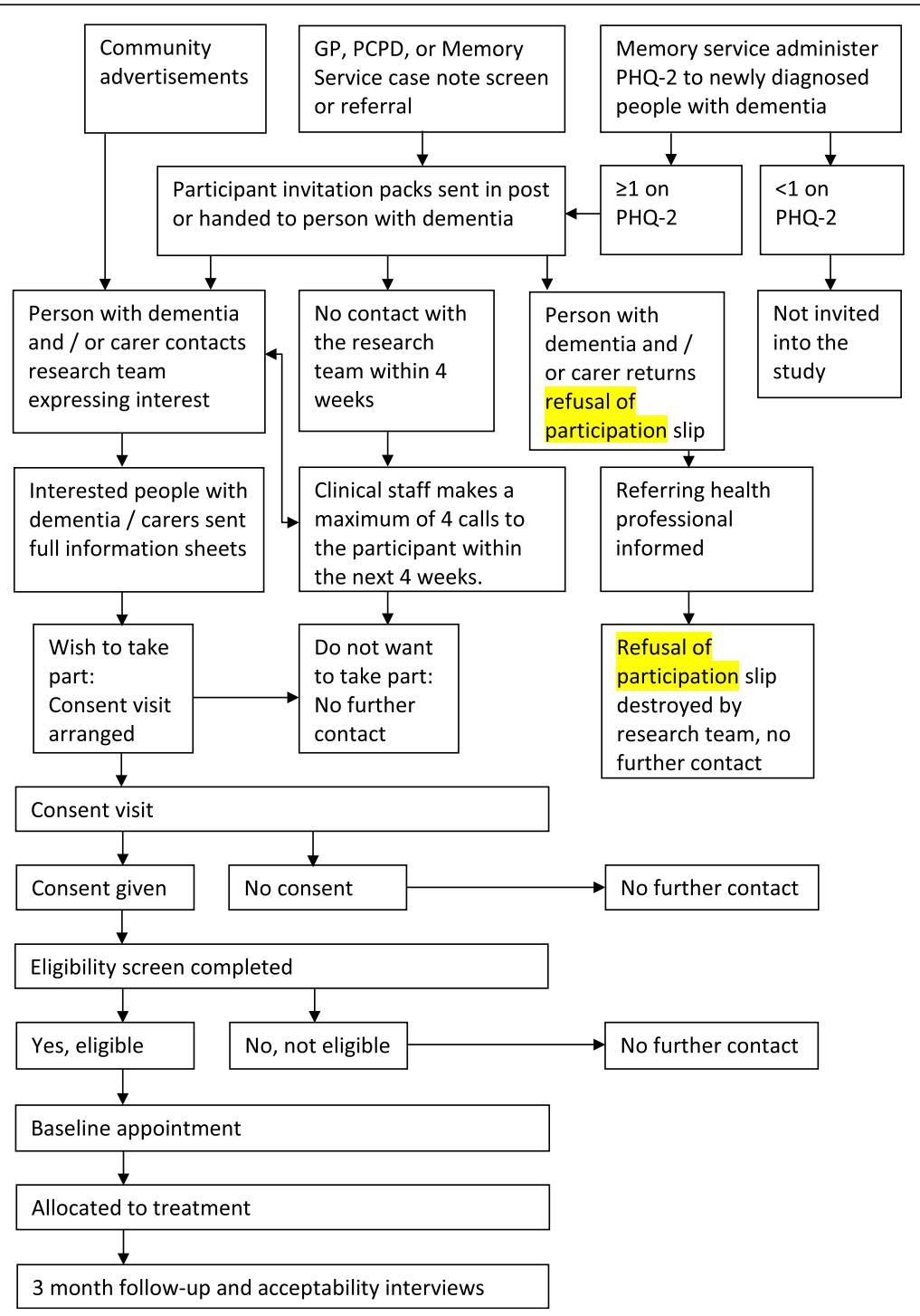

Fig. 1 Flow of participants through the study

\section{Memory service}

The memory service (Consultant in Old Age Psychiatry and Memory Assessment Nurses) will recruit potential participants using two methods: (1) post-diagnostic assessments with memory service staff administering the PHQ-2, a two-item screen for possible depression $[62,63]$, with people with dementia screening positive for possible depression provided with a study invitation package and (2) a search and screening of the memory service database for people who have received a diagnosis of dementia over the preceding 24 months with those identified as potentially eligible being sent the study invitation package in the post. In the case of post-diagnostic assessments only, the administration of the PHQ-2 screen is included within the recruitment procedure to help minimise the number of people invited into the study who do not experience low mood or depression. Post-diagnostic assessments are conducted by health professionals experienced in screening for depression (Consultant in Old Age Psychiatry and Memory Assessment Nurses) and are the only consistent face-to-face recruitment strategy utilised in the study. As such, administration of the PHQ-2 screen will only take place in this recruitment centre. Other faceto-face recruitment techniques (e.g., GP or PCDP referral) are designed to be more opportunistic and time limited, as such the addition of a PHQ-2 screen was not deemed necessary. Given that depression status may have changed since post-diagnostic assessment, people with a diagnosis of dementia recorded on the memory service database will be invited to participate in the study whether or not they have a record of depression documented. Memory service staff will provide non-responders with telephone 
reminders (maximum of four attempts). Anonymised reasons for exclusion from both the post-diagnostic assessments and database screen will be provided to the research team.

\section{Community outreach}

A number of community-based organisations including Memory Cafés, Memory Matters South West, the Alzheimer's Society, Carers Forum, Disability Cornwall, Dementia Action Alliance, Age UK, Cornwall Carers and Penwith Community Development Trust will be approached to support the study by distributing flyers and invitation packages and displaying posters. In addition, the study will be advertised by distributing study posters and leaflets in other community locations including community day care centres; libraries, banks, bus stops, post offices, supermarkets, cafes and community centres.

\section{Reasons for refusal of participation}

Across all recruitment settings, study invitation packages will include refusal of participation forms for both the person with dementia and informal carer for those who do not wish to participate. The form includes a questionnaire listing possible reasons for refusal of participation informed by previous research [64] as well as an openended question to provide further reasons if wished. Each package also includes a freepost envelope to enable people with dementia and their carers to return refusal of participation forms to the study team. These data will be used to inform barriers of recruitment and the acceptability of the proposed intervention.

\section{Informed consent, screening and baseline}

A researcher (MA or SV) will speak to all people with dementia and informal carers who respond to the study invitation package or study advertisements expressing interest to participate or seeking more information about the study. A researcher will arrange to visit those people with dementia and informal carers who are interested in participating in the study, face-to-face, to obtain written consent. Following Mental Capacity Act guidance [65], the researchers will assess capacity to consent in people with dementia, whilst providing appropriate support to maximise their ability to provide consent [66]. If the person with dementia is assessed as lacking capacity to consent, an informal carer will be asked to act as a consultee $[65,67]$. Once consent is obtained from the informal carer, they, alongside the person with dementia, will undergo a face-to-face screening assessment with a researcher, in a location identified by the dyad as convenient. If eligibility is confirmed for both, a full baseline assessment will be arranged with the dyad, again in a convenient location. After the full baseline assessment has been conducted, dyads will be allocated to a PWP by a member of the research team, ensuring as best as possible an even balance across PWPs (see Fig. 1).

\section{Sample size}

A primary aim of this study is to examine the recruitment of participants, through a variety of techniques; therefore, the study will not stipulate a sample size a priori. Instead, to facilitate comparisons between the effectiveness of the recruitment approaches adopted, the study will continue to recruit until a maximum cut-off of 50 dyads (100 participants). Fifty dyads will enable the estimation of a follow-up rate of $80 \%$ with a margin of error of $14 \%$ based on the lower bound of the $95 \%$ confidence interval.

\section{Intervention}

\section{Content}

The BA intervention is informed by the simple BA protocol $[38,40]$ originally developed for PWP delivery of lowintensity treatment within the IAPT programme [68].

\section{Materials}

Two workbooks have been developed, one designed for the person with dementia [69] and the other for the informal carer to aid supporting the person with dementia work through the intervention [70]. The workbook for the person with dementia was written in line with national guidance for the development of dementia-friendly written information [71] describing steps of the BA intervention and providing accompanying worksheets. The informal carer workbook provides guidance on how to support the person with dementia implement the steps involved within the BA protocol, alongside additional support to manage in the caring role, informed by an existing self-help intervention for carers of stroke survivors [72]. To maximise acceptability, the content and design of both workbooks were further informed by a series of qualitative studies with people with dementia and their informal carers.

\section{Support}

A PWP will guide the use of the BA self-help programme, providing a maximum of 12 sessions over 3 months (see Table 1 for the treatment support protocol). These 12 sessions will include one initial assessment session, one setting up support session, up to nine brief telephone support 'check-ins' representing minimal contact support $[26,73]$ to the informal carer and finally, one relapse prevention session. PWPs will follow a structured support protocol, adapted from existing support protocols for PWP assessment and support sessions [68] and brief telephone support sessions [75]. 
Table 1 Treatment support protocol

\begin{tabular}{|c|c|c|c|c|}
\hline Session number & Attendees & Method of support & Session content & Session duration (min) \\
\hline 1 & $\begin{array}{l}\text { Person with dementia; } \\
\text { informal carer; PWP }\end{array}$ & Face-to-face & $\begin{array}{l}\text { Problem focused assessment to identify the } \\
\text { main difficulties with mood and wellbeing } \\
\text { experienced by the person with dementia } \\
\text { and to introduce the BA approach. The } \\
\text { informal carer will also be present to act as } \\
\text { an informant if required. }\end{array}$ & 50 \\
\hline 2 & $\begin{array}{l}\text { Person with dementia; } \\
\text { informal carer; PWP }\end{array}$ & Face-to-face & $\begin{array}{l}\text { A setting up support session to help } \\
\text { establish the protocol for supporting the BA } \\
\text { self-help intervention. The rationale for the } \\
\text { BA intervention is discussed alongside the } \\
\text { procedure for the carer to provide on-going } \\
\text { support to the person with dementia. }\end{array}$ & 40 \\
\hline 3-11 (maximum) & Informal carer; PWP & Telephone & $\begin{array}{l}\text { Minimal contact telephone 'check-ins' to } \\
\text { check progress made with the intervention, } \\
\text { problem solve any difficulties experienced } \\
\text { with using the workbook, agree next steps } \\
\text { and provide on-going encouragement in } \\
\text { the use of the BA self-help workbooks. }\end{array}$ & Up to 15 \\
\hline 12 & $\begin{array}{l}\text { Person with dementia; } \\
\text { informal carer; PWP }\end{array}$ & Face-to-face & $\begin{array}{l}\text { Relapse prevention and provision of } \\
\text { information to enhance on-going } \\
\text { signposting to appropriate health and } \\
\text { social care organisations as needed. }\end{array}$ & 40 \\
\hline
\end{tabular}

\section{Setting}

All participants will be treated within the 'BeMe' primary care mental health service commissioned under the IAPT programme [29] and part of Cornwall Partnership NHS Foundation Trust. All face-to-face sessions will be offered in BeMe offices, community settings or the participants' home if necessary to ensure inclusion into the study for all those wishing to participate.

\section{PWPs}

PWPs will be existing employees of the IAPT primary care mental health service supporting the study. Initially, three existing staff members will be asked to support the study by the service clinical lead, with additional PWPs trained to support the study should capacity need to be increased. All PWPs supporting the intervention will have to successfully complete the IAPT PWP training programme [68] to support the delivery of CBT self-help interventions. Additionally, a 2-day training session specific to supporting the new intervention will be delivered. Group-based clinical supervision to all PWPs supporting the intervention will be provided once a month by the principal investigator, PF.

\section{PWP adherence}

With participant consent, all assessment and support sessions will be recorded. To assess adherence to the support protocol, the marking criteria used as the basis of competency assessments within the IAPT training programme for PWPs will be adopted [68]. Necessary adaptations to the support protocol and associated marking criteria have been undertaken to ensure the low-intensity CBT clinical method is consistent and appropriate for use in the study with people with dementia and informal carers. A sample of $20 \%$ of sessions will be randomly selected for each PWP, and adherence to the support protocol will be judged by a clinician otherwise not associated with the study. This clinician will be considered competent to mark the tapes by virtue of having completed and passed the IAPT programme for the training for PWPs themselves and having undertaken additional training with the research team in the use of the marking criteria and adaptations for a dementia population.

\section{Feasibility outcome measurements (primary outcome measurements)}

Data related to study feasibility will be collected to examine the primary study objectives relating to methodological, procedural and clinical uncertainties $[47,48]$. Following guidance concerning feasibility study objectives [47], Table 2 provides a summary of feasibility outcomes to be examined, alongside progression criteria (where applicable) to be met in order to progress to develop an application for phase II pilot RCT funding [45].

\section{Clinical outcome measurements (secondary outcome measurements)}

To examine the feasibility of the data collection procedures, a number of clinical outcome measures will be collected from people with dementia and their informal carers (see Table 3). 
Table 2 Feasibility data and method of measurement

\begin{tabular}{|c|c|c|}
\hline Feasibility outcome & Measurement & Progression criteria to phase II pilot RCT \\
\hline \multirow[t]{12}{*}{ Recruitment } & Quantitative data & \\
\hline & $\begin{array}{l}\text { Percentage of people with dementia invited into the study/number of people } \\
\text { with dementia in total identified by health professionals }\end{array}$ & No criteria set \\
\hline & Number of health professionals required to assist with recruitment into the study & No criteria set \\
\hline & The time taken (up to 6 months) to recruit up to 50 dyads & No criteria set \\
\hline & Number of dyads enrolled into the study per week & 2 dyads per week \\
\hline & $\begin{array}{l}\text { Percentage of dyads willing to undergo screening/number invited (calculated for } \\
\text { GP, PCPD and memory service recruitment) }\end{array}$ & $\geq 15 \%$ \\
\hline & Percentage of dyads overall meeting the inclusion criteria/number invited & $\geq 5 \%$ \\
\hline & Percentage of dyads overall enrolled in the study/number invited & $\geq 5 \%$ \\
\hline & Qualitative data & \\
\hline & $\begin{array}{l}\text { Reasons for exclusion reported to the research team during health professional } \\
\text { screening (GP, PCPDs, memory service) }\end{array}$ & No criteria set \\
\hline & Reasons for ineligibility & No criteria set \\
\hline & Identified barriers to recruitment (reasons for refusal of participation) & No criteria set \\
\hline \multirow[t]{5}{*}{ Attrition } & Quantitative data & \\
\hline & Percentage of dyads completing post-treatment (3 month) outcome measures & $\geq 70 \%$ \\
\hline & Reasons for dropout & No criteria set \\
\hline & Qualitative data & \\
\hline & $\begin{array}{l}\text { Acceptability interviews with non-attendees and poor attendees (informal carers } \\
\text { and people with dementia) }\end{array}$ & No criteria set \\
\hline \multirow{8}{*}{$\begin{array}{l}\text { Data collection } \\
\text { procedures }\end{array}$} & Quantitative data & \\
\hline & Time taken and number of sessions to administer the screening measures & $\leq 2 \mathrm{~h} ; \leq 2$ sessions \\
\hline & Time taken and number of sessions to administer the baseline assessment & $\leq 2 \mathrm{~h} ; \leq 2$ sessions \\
\hline & Time taken and number of sessions to administer the follow-up assessments & $\leq 2 \mathrm{~h} ; \leq 2$ sessions \\
\hline & Percentage of missing items per questionnaire & $\leq 10 \%$ \\
\hline & Qualitative data & \\
\hline & $\begin{array}{l}\text { Acceptability interviews with participants concerning acceptability of research } \\
\text { procedures }\end{array}$ & No criteria set \\
\hline & $\begin{array}{l}\text { Acceptability interviews with PWPs concerning acceptability and feasibility of } \\
\text { research procedures }\end{array}$ & No criteria set \\
\hline Clinician adherence & Adherence to support protocol as determined by therapy tapes & $\geq 70 \%$ \\
\hline \multirow[t]{12}{*}{ Clinical delivery } & Quantitative data & \\
\hline & $\begin{array}{l}\text { Time between being allocated to PWP and PWP undertaking the assessment } \\
\text { session. }\end{array}$ & $\leq 2$ weeks \\
\hline & Session lengths & No criteria set \\
\hline & Number of sessions received per dyad & No criteria set \\
\hline & Settings of sessions (e.g., BeMe, community, home) & No criteria set \\
\hline & Number of missed appointments & No criteria set \\
\hline & Number of missed outcome measurement items & No criteria set \\
\hline & PWP attrition & No criteria set \\
\hline & $\begin{array}{l}\text { Impact of severity of dementia (MMSE score) informing who can engage in the } \\
\text { intervention }\end{array}$ & No criteria set \\
\hline & Qualitative data & \\
\hline & Acceptability interviews with participants & No criteria set \\
\hline & Acceptability interviews with PWPs & No criteria set \\
\hline
\end{tabular}


Table 3 Study clinical outcome measurements by time point

\begin{tabular}{ll}
\hline Outcome measure & Time point \\
\hline Person with dementia & \\
Sociodemographics & Initial screen \\
MMSE [56] & Initial screen \\
GDS-12R [57] & Initial screen, post-treatment follow-up \\
CSDD [76] & Baseline, post-treatment follow-up \\
DEMQOL [81] & Baseline, post-treatment follow-up \\
EQ-5D-3L [82] & Baseline, post-treatment follow-up \\
Informal carer & \\
Sociodemographics & Initial screen \\
PHQ-9 [84] & Initial screen, post-treatment follow-up \\
GAD-7 [85] & Baseline, post-treatment follow-up \\
ZBI-12 [86] & Baseline, post-treatment follow-up \\
CSDD-proxy [76] & Baseline, post-treatment follow-up \\
SF-12 [87] & Baseline, post-treatment follow-up \\
EQ-5D-3L [83] & Baseline, post-treatment follow-up \\
Revised CSRI [88] & Baseline, post-treatment follow-up \\
\hline
\end{tabular}

MMSE mini-mental state examination, GDS-12R Geriatric Depression Scale-12 Residential, CSDD Cornell Scale for Depression in Dementia, DEMQOL Dementia Quality of Life Measure, EQ-5D-3L EuroQol-5D-3L, PHQ-9, Health Questionnaire-9, GAD-7 Generalised Anxiety Disorder 7-Item Scale, ZBI-12 Zarit Caregiver Burden Interview Short Form, SF-12 12-Item Short Form Health Survey, CSRI Client Service Receipt Inventory

\section{People with dementia}

The Cornell Scale for Depression in Dementia (CSDD) [76] will be used to assess severity of depressive symptoms, alongside the GDS-12R [48]. The CSDD [71] will be used to assess severity of depressive symptoms, alongside the GDS-12R [57, 77]. The CSDD is an interview-based questionnaire conducted with both the person with dementia and an informal carer as a proxy measure and demonstrated to be a reliable and valid measure for people with both mild and moderate-to-severe levels of dementia $[78,79]$. The GD-12R is reliable for people with moderate-to-severe levels of dementia $[57,77]$ and recommended for use in psychosocial intervention research for people with dementia [80]. Quality of life will be examined through the Dementia Quality of Life measure [81] and the EuroQol-5D-3L (EQ-5D-3L) [82] validated in a mild-to-moderate dementia population [83].

\section{Informal carers}

The PHQ-9 [84] and the Generalised Anxiety Disorder Questionnaire (GAD-7) [85] will be administered to assess severity of depressive and anxious symptoms, respectively. Carer burden will be measured through the administration of the Zarit Caregiver Burden Interview Short Form (ZBI-12) [86]. Quality of life will be assessed through the Medical Outcome Survey Short Form 12 (SF-12) [87] and EQ-5D-3L [82]. Health and social care use, for both the person with dementia and informal carer, will be collected via the administration of an adapted version of the Client Socio-Demographic and Service Receipt Inventory (CSRI) [88], based on two versions developed for carers of stroke survivors [74, 89]. In addition, the CSDD-proxy [76] will be administered to informal carers to collect further information concerning the severity of depressive symptoms in the person with dementia.

\section{Sociodemographics and clinical characteristics}

During the initial screen, various background, clinical and socio-demographic characteristics-age, gender, ethnic background, relationship status, employment status, highest level of academic qualification, yearly household income, chronic physical health conditions-will be collected from people with dementia and informal carers. This will be supplemented by the additional collection of dementia subtype diagnosed and date of diagnosis from the person with dementia. Informal carers will also be asked to confirm the dementia subtype diagnosed and date of diagnosis as well as yearly household income (if the carer does not live with the person with dementia), length of time caring, if the carer lives with the person with dementia, provision of care before diagnosis of dementia, receipt of support services in the home, hours of support services received in the home per week and hours of caring per week.

\section{Data collection}

Researchers will collect data from the person with dementia and informal carer face-to-face, at a location convenient for the person with dementia and informal carer. To protect confidentiality, each dyad will be offered the choice of completing outcome measurements at an alternative faceto-face appointment to the other member of the dyad should they prefer. Data will be collected at screening, baseline, and post-treatment (3 months post-treatment allocation). Clinical outcome measurements collected at each time point are summarised in Table 3.

\section{Intervention acceptability \\ Study objectives and design}

An embedded qualitative study will be conducted to determine the views of people with dementia and informal carers regarding the acceptability of the BA self-help intervention. PWPs supporting the intervention will be interviewed with respect to the acceptability and feasibility of the BA self-help intervention and support protocol. All people with dementia and informal carers will be invited to participate in semi-structured face-to-face or telephonebased interviews using open-ended questions addressing:

1. Relevance of the intervention

2. Suitability of the intervention 
3. Impressions of guidance provided by PWPs

4. Perceived benefit of the intervention

5. Problems experienced utilising the intervention

6. Continued use of the intervention

7. Recommendations for further treatment development

8. Acceptability of research processes

The interview topic guide is informed by interview topic guides for CBT self-help for patients with multiple sclerosis [90] and carers of stroke survivors [74]. Informal carers and those with dementia who have poor session attendance will be invited to take part in an interview session which will ask questions concerning the reasons for disengaging with the intervention and recommendations for a more acceptable intervention. Interviews are estimated to last between 30 and $60 \mathrm{~min}$, with shorter times expected for non/poor attendees.

Semi-structured interviews with PWPs will be conducted over the telephone and are expected to last between 45 and $60 \mathrm{~min}$. Open-ended questions will be asked about the PWP's impressions of the following:

1. The self-help intervention

2. Difficulties or problems encountered providing support

3. Recommendations for future development of the intervention and PWP training

4. Acceptability and feasibility of collecting in-session outcome measurements

5. Acceptability and feasibility of research processes

\section{Sampling}

All participants will be invited to participate in the acceptability interviews. The research team will attempt to interview participants categorised into one of the following groups: (1) non-attendees (no sessions attended); (2) poor attendees (participants who attend the assessment session but terminate treatment prior to making a collaborative decision with the PWP to stop treatment); or (3) completers (engagement in treatment until a collaborative decision with the PWP to stop treatment is made). All study PWPs will be interviewed concerning the acceptability of the intervention.

\section{Statistical analysis \\ Quantitative}

Recruitment data: where possible, data will be collected separately from each recruitment setting to determine recruitment rate and methodological uncertainties associated with each specific setting. Data will be collected relating to the type and number of referring sites; number of participants identified at each site; number of invites sent (where possible, this will not be possible with community recruitment), number of potential participants requesting more information, number of consents obtained, number of screens completed, number of baselines completed and number of participants allocated to a PWP. Number of exclusions at each stage and reasons for exclusions will also be reported. The percentage of eligible participants that are recruited will be calculated with exact $95 \%$ confidence intervals using the cii command in Stata software version 14.0.

Feasibility of data collection procedures and acceptability to patients: study protocol deviations along with reasons will be reported to assess both the feasibility and acceptability of the data collection process. The time taken to administer the screening measures, time taken to administer the baseline assessment, time taken to administer the follow-up assessments and the percentage of missing data per outcome measurement collected will also be reported to examine the feasibility and acceptability of data collection procedures.

Attrition (study dropout): the number of participants dropping out of the study will be reported, along with the stage of dropout and reasons where possible. Attrition proportions will be reported with $95 \%$ confidence intervals.

Primary and secondary outcome measurements: descriptive statistics including the means and standard deviations or medians and interquartile ranges will be reported for each outcome measurement at baseline and 3 months post-treatment allocation as these will help to inform the sample size for the pilot RCT and later definitive trial.

\section{Qualitative}

A thematic analysis approach [91] will be adopted to analyse transcribed digital recordings from the interviews. To ensure rigour $[92,93]$ and confirm consistency with generated themes, two members of the research team (MA, SV) will analyse each interview separately with analyses then compared. Two other research team members (JW and PF), alongside members of the lived experience steering committee (details below), will discuss a subset of the analysed interviews to additionally ensure the analysis reflects the generated themes.

\section{Lived experience steering committee}

A lived experience steering committee has been established as research collaborators [94], consisting of two people with dementia and two informal carers (one spouse and one adult child carer of their mother). Specifically, the lived experience steering committee is responsible for assisting with research activities such as developing accessible participant materials, advising on recruitment and research procedures and supporting PWP training and analysis of the qualitative data. The steering committee will aim to meet monthly throughout the course of the study as active members of the wider research team. 


\section{Dissemination}

Findings of the study will be published in an open access journal and via conference presentation. The data from this study will be used to inform the design and accompanying grant application for a future pilot RCT should the study be considered feasible with set progression criteria met.

\section{Discussion}

To the best of our knowledge, this feasibility study represents the first investigation into the feasibility and acceptability of a written BA-based self-help intervention for the treatment of depression in people with dementia. A BA self-help intervention, guided by PWPs and supported by informal carers has the potential to represent an effective, acceptable and cost-effective solution to address the significant unmet need in the provision of psychological support for people with dementia. Furthermore, equipping informal carers with strategies to help improve the wellbeing of care recipients may also improve mood and reduce burden in the carers themselves [36]. This current study will explore important questions pertaining to the acceptability and feasibility of the new intervention and research procedures.

Should the current study demonstrate feasibility and achieve the progression criteria specified, outcomes will be used to design and inform a funding application for a phase II pilot RCT [45]. The aim of this pilot RCT will be to specifically test trial processes associated with an RCT design, with results used to develop and inform a funding application for a future definitive phase III [45] trial to examine effectiveness.

\section{Study status}

Study recruitment will commence in January 2016. The final outcome data will be collected in September/ October 2016.

\begin{abstract}
Abbreviations
BA, behavioural activation; CSRI, Client Socio-Demographic and Service Receipt Inventory; CBT, cognitive behavioural therapy; CSDD, Cornell Scale for Depression in Dementia; GAD-7, Generalised Anxiety Disorder Questionnaire; GDS, Geriatric Depression Scale; IAPT, Improving Access to Psychological Therapies; MRC, Medical Research Council; MMSE, mini-mental state examination; PHQ-9, Patient Health Questionnaire-9; PWP, Psychological Wellbeing Practitioner; RCT, randomised controlled trial; SF-12, Medical Outcome Survey Short Form; SPIRIT, Standard Protocol Items, Recommendations for Interventional Trials; ZBI-12, Zarit Caregiver Burden Interview Short Form
\end{abstract}

\section{Acknowledgements}

We are grateful to our Lived Experience Committee as well as Clare Jeckells, Sarah Twomlow and Mary Williamson (study PWPS). We are also grateful for the support provided by Dr. Joe Ryan, service lead for the BeMe IAPT Service and the Complex Care and Dementia Community team within Cornwall Foundation Partnership Trust. Obioha Ukoumunne is supported by the National Institute for Health Research (NIHR) Collaboration for Leadership in Applied Health Research and Care South West Peninsula. The views expressed are those of the author(s) and not necessarily those of the NHS, the NIHR or the Department of Health.

\section{Funding}

This study is collaboratively funded by Cornwall Foundation Partnership Trust, South West Peninsula Academic Health Sciences Network and the University of Exeter.

\section{Authors' contributions}

PF contributed to the conception and design of the protocol, wrote the manuscript and gave final approval of the manuscript. JW contributed to the design of the protocol, wrote the study protocol with oversight from PF, wrote the manuscript and gave final approval of the manuscript. MA contributed to the data collection and critical revision and gave final approval of the manuscript. SV contributed to the data collection and critical revision and gave final approval of the manuscript. DL contributed to the critical revision and gave final approval of the manuscript. OU contributed to the critical revision and gave final approval of the manuscript. AA contributed to the critical revision and gave final approval of the manuscript. CD contributed to the critical revision and gave final approval of the manuscript.

\section{Competing interests}

The authors declare they have no competing interests.

\section{Ethics approval and consent to participate}

The study will be conducted in accordance with the Helsinki Declaration to safeguard the welfare and rights of participants. Ethical approval was received by the National Research Ethics Committee London-South East on 16/11/2015, REC Reference number: 15/LO/1689. In a manner consistent with the Data Protection Act, all research data will be stored without participant identifiers, in a secure Access databases on a password-protected computer at the University of Exeter, only accessible by the research team. To ensure data quality, all outcome data will be entered in duplicate by two researchers. Participant names and addresses will be stored on a separate secure Access database, and all paper-based data will be stored in a locked filing cabinet.

\section{Sponsors}

Professor Paul Farrand is the principal investigator and conceived the intervention and the study design and is providing on-going management of the study. The University of Exeter is acting as the study sponsor.

\section{Author details}

${ }^{1}$ Clinical Education Development and Research (CEDAR), Psychology: College of Life and Environmental Sciences, University of Exeter, Washington Singer Labs, Perry Road, Exeter EX4 4QG, UK. ²University of Exeter Medical School, St. Luke's Campus, Exeter EX1 2LU, UK. ${ }^{3} \mathrm{NIHR}$ CLAHRC South West Peninsula, University of Exeter Medical School, St. Luke's Campus, Exeter EX1 2LU, UK.

Received: 19 December 2015 Accepted: 25 June 2016

Published online: 04 August 2016

References

1. Prince M, Bryce R, Albanese E, Wimo A, Riberio W, Ferri CP. The global prevalence of dementia: a systematic review and metaanalysis. Alzheimers Dement. 2013;9:63-75.

2. Ritchie CW, Terrera GM, Quinn TJ. Dementia trials and dementia tribulations: methodological and analytical challenges in dementia research. Alzheimers Res Ther. 2015;7:31.

3. Wu YT, Fratiglioni L, Matthews FE, Lobo A, Breteler MM, Skoog I, Brayne C. Dementia in western Europe: epidemiological evidence and implications for policy making. Lancet Neurol. 2016; 15(1):116-124.

4. Matthews FE, Arthur A, Barnes LE, Bond J, Jagger C, Robinson L, Brayne C. A two-decade comparison of prevalence of dementia in individuals aged 65 years and older from three geographical areas of England: results of the Cognitive Function and Ageing Study I and II on behalf of the Medical Research Council Cognitive Function and Ageing Collaboration. Lancet. 2013;382:1405-12.

5. Clare L, Nelis SM, Quinn C, Martyr A, Henderson C, Hindle JV, et al. Improving the experience of dementia and enhancing active life-living well with dementia: study protocol for the IDEAL study. Health Qual Life Outcomes. 2014;12:164. 
6. Department of Health. Prime minister's challenge on dementia: delivering major improvements in dementia care and research by 2015. London: Department of Health; 2012.

7. Samsi K, Manthorpe J. Care pathways for dementia: current perspectives. Clin Interv Aging. 2014;9:2055-63.

8. Mathers C, Boerma T, Fat DM. The global burden of disease: 2004 update. Geneva: World Health Organization; 2008.

9. Pattanyak RD, Sagar R. Depression in dementia patients: issues and challenges for a physician. J Assoc Physicians India. 2011;59:650-2.

10. Bennett S, Thomas AJ. Depression and dementia: cause, consequence or coincidence? Maturitas. 2014;79:184-90.

11. Enache D, Winblad B, Aarsand D. Depression in dementia: epidemiology, mechanisms and treatment. Curr Opin Psychiatry. 2011;24:461-72.

12. Orgeta V, Qazi A, Spector AE, Orrell M. Psychological treatments for depression and anxiety in dementia and mild cognitive impairment. Cochrane Database Syst Rev. 2014;1:CD009125

13. Modrego PJ. Depression in Alzheimer's disease. Patho-physiology, diagnosis, and treatment. J Alzheimers Dis. 2010;21:1077-87.

14. Novaisa F, Starkstein S. Phenomenology of depression in Alzheimer's disease. J Alzheimers Dis. 2015;47:845-55.

15. van der Roest HG, Meiland FJ, Comijs HC, Derksen E, Jansen AP, van Hout HP, et al. What do community-dwelling people with dementia need? A survey of those who are known to care and welfare services. Int Psychogeriatr. 2009;21:949-65.

16. Kohn R, Saxena S, Levav I, Saraceno B. The treatment gap in mental health care. Bull World Health Organ. 2004;82:858-66.

17. Kazdin AE, Blase SL. Rebooting psychotherapy research and practice to reduce the burden of mental illness. Perspect Psychol Sci. 2011;6:21-37.

18. Lovell $K$, Richards DA, Bower P. Improving access to primary mental health care: uncontrolled evaluation of a pilot self-help clinic. Br J Gen Pract. 2003:53:133-5.

19. Wittchen HU, Jacobi F, Rehm J, Gustavsson A, Svensson M, Jönsson B, et al. The size and burden of mental disorders and other disorders of the brain in Europe 2010. Eur Neuropsychopharmacol. 2011;21:655-79.

20. Gyani A, Shafran R, Layard R, Clark DM. Enhancing recovery rates: lessons from year one of IAPT. Behav Res Ther. 2013:51:597-606.

21. Pilgrim D, Carey TA. Improving access to psychological therapies: an account of recent policy aspirations in the UK and Australia. Adv Menta HIth. 2012;10:117-26

22. Rebello TJ, Marques A, Gureje O, Pike KM. Innovative strategies for closing the mental health treatment gap globally. Curr Opin Psychiatry. 2014;27: 308-14.

23. Vis C, Kleibor A, Prior R, Bønes E, Cavallo M, Clark S, et al. Implementing and up-scaling evidence-based eMental health in Europe: the study protocol for the MasterMind project. Internet Interv. 2015;2:339-409.

24. Ridgway N, Williams C. Cognitive behavioural therapy self-help for depression: an overview. J Ment Health. 2011:20:593-603.

25. Donker T, Petrie K, Proudfoot J, Clarke J, Birch MR, Christensen H. Smartphones for smarter delivery of mental health programs: a systematic review. J Med Internet Res. 2013;15:e247.

26. Farrand P, Woodford J. Impact of support on the effectiveness of written cognitive behavioral self-help: a systematic review and meta-analysis of randomized controlled trials. Clin Psychol Rev. 2013;33:182-95.

27. Andersson G, Cuijpers P. Internet-based and other computerized psychological treatments for adult depression: a meta-analysis. Cogn Behav Ther. 2009;38:196-205

28. Gellatly S, Bower P, Hennessy S, Richards DA, Gilbody S, Lovell K. What makes self-help interventions effective in the management of depressive symptoms? Meta-analysis and meta-regression. Psychol Med. 2007;37:1217-28.

29. Clark DM. Implementing NICE guidelines for the psychological treatment of depression and anxiety disorders: the IAPT experience. Int Rev Psychiatry. 2011:23:318-27.

30. Bower P, Gilbody S. Stepped care in psychological therapies: access, effectiveness and efficiency. Narrative literature review. Br J Psychiatry. 2005;186:11-7

31. McClay CA, Collins K, Matthews L, Haig C, McConnachie A, Morrison J, et al. A community-based pilot randomised controlled study of life skills classes for individuals with low mood and depression. BMC Psychiatry. 2015;15:17.

32. Carretero S, Garcés J, Ródenas F, Sanjosé V. The informal caregiver's burden of dependent people: theory and empirical review. Arch Gerontol Geriatr. 2009;49:74-9.
33. Ask H, Langballe EM, Holmen J, Selbæk G, Saltvedt I, Tambs K. Mental health and wellbeing in spouses of persons with dementia: the Nord-Trøndelag health study. BMC Public Health. 2014;14:1.

34. Mausbach BT, Chattillion EA, Moore RC, Roepke SK, Depp CA, Roesch S. Activity restriction and depression in medical patients and their caregivers: a meta-analysis. Clin Psychol Rev. 2011;31:900-8.

35. Thomas P, Lalloué F, Preux PM, Hazif-Thomas C, Pariel S, Inscale R, Belmin J, Clément JP. Dementia patients caregivers quality of life: the PIXEL study. Int J Geriatr Psychiatry. 2006;21:50-6.

36. Teri L, Logsdon RG, Uomoto J, McCurry SM. Behavioral treatment of depression in dementia patients: a controlled clinical trial. J Gerontol B Psychol Sci Soc Sci. 1997:52:159-66.

37. Farrand P, Pentecost C, Greaves C, Taylor RS, Warren F, Green C, et al. A written self-help intervention for depressed adults comparing behavioural activation combined with physical activity promotion with a self-help intervention based upon behavioural activation alone: study protocol for a parallel group pilot randomised controlled trial (BACPAC). Trials. 2014;15:196

38. Ekers D, Webster L, van Straten A, Cuijpers P, Richards D, Gilbody S. Behavioural activation for depression; an update of meta-analysis of effectiveness and sub group analysis. PLoS One. 2014;17:e100100.

39. Ekers D, Godfrey C, Gilbody SM, Parrott SM, Richards DA, Hammond D, Hayes A. Cost utility of behavioural activation delivered by the nonspecialist. Br J Psychiatry. 2011;199:510-1.

40. Richards D. Behavioural Activation. In: Bennett-Levy J et al., editors. In Oxford guide to low intensity CBT interventions. Oxford: Oxford University Press; 2011. p. 141-50

41. Dimidjian S, Hollon SD, Dobson KS, Schmaling KB, Kohlenberg RJ, Addis ME, et al. Randomized trial of behavioural activation, cognitive therapy, and antidepressant medication in the acute treatment of adults with major depression. J Consult Clin Psychol. 2006;74:658-70.

42. Toms GR, Quinn C, Anderson DE, Clare L. Help yourself: perspectives on self-management from people with dementia and their caregivers. Qual Health Res. 2015;25:87-98.

43. Teri L, McCurry SM, Logsdon R, Gibbons LE. Training community consultants to help family members improve dementia care: a randomized controlled trial. Gerontologist. 2005:45:802-11.

44. Besdine R, Boult C, Brangman S, Coleman EA, Fried LP, Gerety M, et al. Caring for older Americans: the future of geriatric medicine. J Am Geriatr Soc. 2005:53:S245-56.

45. Craig P, Dieppe P, Macintyre S, Michie S, Nazareth I, Petticrew M, et al. Developing and evaluating complex interventions: the new Medical Research Council guidance. BMJ. 2008;337:A1655.

46. McKeown J, Clarke A, Ingleton C, Repper J. Actively involving people with dementia in qualitative research. J Clin Nurs. 2010:19:1935-43.

47. Abbott JH. The distinction between randomized clinical trials (RCTs) and preliminary feasibility and pilot studies: what they are and are not. J Orthop Sports Phys Ther. 2014;44:555-8.

48. Thabane L, Ma J, Chu R, Cheng J, Ismaila A, Rios LP, et al. A tutorial on pilot studies: the what, why and how. BMC Med Res Methodol. 2010;10:1

49. Chan AW, Tetzlaff JM, Gøtzsche PC, Altman DG, Mann H, Berlin JA, Dickersin K, Hróbjartsson A, Schulz KF, Parulekar WR, Krleža-Jerić K. SPIRIT 2013 explanation and elaboration: quidance for protocols of clinical trials. BMJ. 2013;346:e7586.

50. Kuyken W, Byford S, Byng R, Dalgleish T, Lewis G, Taylor R, et al. Study protocol for a randomized controlled trial comparing mindfulness-based cognitive therapy with maintenance anti-depressant treatment in the prevention of depressive relapse/recurrence: the PREVENT trial. Trials. 2010;11:99.

51. Richards DA, Hughes-Morley A, Hayes RA, Araya R, Barkham M, Bland JM, et al. Collaborative Depression Trial (CADET): multi-centre randomised controlled trial of collaborative care for depression-study protocol. BMC Health Serv Res. 2009;9:188.

52. Watkins ER, Mullan E, Wingrove J, Rimes K, Steiner $H$, Bathurst N, et al. Rumination focused cognitive-behavioural therapy for residual depression: phase II randomised controlled trial. Br J Psychiatry. 2011;199:317-22.

53. Spector A, Orrell M, Lattimer M, Hoe J, King M, Harwood K, Qazi A, Charlesworth G. Cognitive behavioural therapy (CBT) for anxiety in people with dementia: study protocol for a randomised controlled trial. Trials. 2012;13:197.

54. Whitebird RR, Kreitzer MJ, Lewis BA, Hanson LR, Crain AL, Enstad CJ, Mehta A. Recruiting and retaining family caregivers to a randomized controlled trial on mindfulness-based stress reduction. Contemp Clin Trials. 2011:32:654-61.

55. Schneider JA, Arvanitakis Z, Bang W, Bennett DA. Mixed brain pathologies account for most dementia cases in community-dwelling older persons. Neurology. 2007;69:2197-204. 
56. Folstein MF, Folstein SE, McHugh PR. "Mini-mental state". A practical method for grading the cognitive state of patients for the clinician. J Psychiatr Res. 1975;12:189-98.

57. Sutcliffe C, Cordingley L, Burns A, Mozley CG, Bagley H, Huxley P, Challis D. A new version of the geriatric depression scale for nursing and residential home populations: the geriatric depression scale (residential) (GDS-12R). Int Psychogeriatr. 2000;12:173-81

58. National Institute for Health and Clinical Excellence. Depression: treatment and management of depression in adults: clinical guideline 90. London: NICE; 2009.

59. Cameron IM, Cardy A, Crawford JR, du Toit SW, Hay S, Lawton K, et al. Measuring depression severity in general practice: discriminatory performance of the PHQ-9, HADS-D, and BDI-II. Br J Gen Pract. 2011;61:e419-26.

60. Jenkinson CE, Winder RE, Sugg HV, Roberts MJ, Ridgway N, Kuyken W, et al. Why do GPs exclude patients from participating in research? An exploration of adherence to and divergence from trial criteria. Fam Pract. 2014;31:364-70.

61. Watson JW, Torgerson DJ. Increasing recruitment to randomised trials: a review of randomised controlled trials. BMC Med Res Methodol. 2006;6:34.

62. Kroenke K, Spitzer RL, Williams JB. The patient health questionnaire-2: validity of a two-item depression screener. Med Care. 2003;41:1284-92.

63. Whooley MA, Avins AL, Miranda J, Browner WS. Case-finding instruments for depression. Two questions are as good as many. J Gen Intern Med. 1997:12:439-45.

64. Barnes M, Wiles N, Morrison J, Kessler D, Williams C, Kuyken W, et al. Exploring patients' reasons for declining contact in a cognitive behavioural therapy randomised controlled trial in primary care. Br J Gen Pract. 2012:62:e371-7.

65. Department for Constitutional Affairs. Mental Capacity Act 2005: code of practice. London: The Stationery Office; 2007.

66. Warner J, McCarney R, Griffin M, Hill K, Fisher P. Participation in dementia research: rates and correlates of capacity to give informed consent. J Med Ethics. 2008:34:167-70.

67. Hope T, Slowther A, Eccles J. Best interests, dementia and the Mental Capacity Act (2005). J Med Ethics. 2009;35:733-8.

68. Richards D, Whyte M. Reach out: national programme support materials to support the delivery of training for psychological wellbeing practitioners delivering low intensity interventions. London: Rethink Mental Illness; 2008.

69. Farrand P, Woodford J, Anderson M, Lovis L. Getting more out of every day with memory difficulties: a guide for people living with memory difficulties. Exeter: Exeter University; 2015.

70. Farrand P, Woodford J, Anderson M. Getting more out of every day with memory difficulties: a guide for family and friends. Exeter: Exeter University; 2015.

71. Dementia Engagement and Empowerment Project (DEEP). Writing dementia-friendly information. Swindon; 2013. http://dementiavoices.org.uk/ wp-content/uploads/2013/11/DEEP-Guide-Writing-dementia-friendlyinformation.pdf.

72. Woodford J, Farrand P. Helping relatives and carers of stroke survivors overcome depression programme. Exeter: Exeter University; 2013.

73. Glasgow RE, Rosen GM. Behavioral bibliotherapy: a review of self-help behavior therapy manuals. Psychol Bull. 1978;85:1-23.

74. Woodford J, Farrand P, Watkins ER, Richards DA, Llewellyn DJ. Supported cognitive-behavioural self-help versus treatment-as-usual for depressed informal carers of stroke survivors (CEDArS): study protocol for a feasibility randomized controlled trial. Trials. 2014;15:157.

75. Williams C, Wilson P, Morrison J, McMahon A, Walker A, Allan L, et al. Guided self-help cognitive behavioural therapy for depression in primary care: a randomised controlled trial. PLoS One. 2013;8:e52735.

76. Alexopoulos GS, Abrams RC, Young RC, Shamoian CA. Cornell scale for depression in dementia. Biol Psychiatry. 1988;23:271-84.

77. Jongenelis K, Pot AM, Eisses AM, Gerritsen DL, Derksen M, Beekman AT, Kluiter H, Ribbe MW. Diagnostic accuracy of the original 30-item and shortened versions of the Geriatric Depression Scale in nursing home patients. Int J Geriatr Psychiatry. 2005;20(11):1067-1074.

78. Kørner A, Lauritzen L, Abelskov K, Gulmann N, Marie Brodersen A, et al. The geriatric depression scale and the cornell scale for depression in dementia. A validity study. Nord J Psychiatry. 2006;60:360-4.

79. Müller-Thomsen T, Arlt S, Mann U, Maß R, Ganzer S. Detecting depression in Alzheimer's disease: evaluation of four different scales. Arch Clin Neuropsychol. 2005:20:271-6.
80. Moniz-Cook E, Vernooij-Dassen M, Woods R, Verhey F, Chattat R, et al. A European consensus on outcome measures for psychosocial intervention research in dementia care. Aging Ment Health. 2008;12:14-29.

81. Smith SC, Lamping DL, Banerjee S, Harwood RH, Foley B, Smith $\mathrm{P}$, et al. Development of a new measure of health-related quality of life for people with dementia: DEMQOL. Psychol Med. 2007;37:737-46.

82. Brooks R. EuroQol: the current state of play. Health Policy. 1996;37:53-72.

83. Hounsome N, Orrell M, Edwards RT. EQ-5D as a quality of life measure in people with dementia and their carers: evidence and key issues. Value Health. 2011;14:390-9.

84. Kroenke K, Spitzer RL, Williams JB. The PHQ-9: validity of a brief depression severity measure. J Gen Intern Med. 2001;16:606-13.

85. Spitzer RL, Kroenke K, Williams JB, Löwe B. A brief measure for assessing generalized anxiety disorder: the GAD-7. Arch Intern Med. 2006;166:1092-7.

86. Bédard M, Molloy DW, Squire L, Dobois S, Lever JA, O'Donnell M. The Zarit Burden interview a new short version and screening version. Gerontologist. 2001:41:652-7.

87. Ware J, Kosinski M, Keller SD. A 12-item short-form health survey: construction of scales and preliminary tests of reliability and validity. Med Care. 1996:34:220-33.

88. Chisholm D, Knapp MR, Knudsen HC, Amaddeo F, Gaite L, van Wijingaarden B. Client Socio-Demographic and Service Receipt Inventory-European version: development of an instrument for international research. EPSILON Study 5. European psychiatric services: inputs linked to outcome domains and needs. Br J Psychiatry Suppl. 2000;39:s28-33.

89. Patel A, Knapp M, Evans A, Perez I, Kalra L. Training care givers of stroke patients: economic evaluation. BMJ. 2004;328:1102-4.

90. Hind D, O'Cathain A, Cooper CL, Parry GD, Isaac CL, Rose A, et al. The acceptability of computerised cognitive behavioural therapy for the treatment of depression in people with chronic physical disease: a qualitative study of people with multiple sclerosis. Psychol Health. 2009;25:699-712.

91. Braun V, Clarke V. Using thematic analysis in psychology. Qual Res Psychol. 2006:3:77-101.

92. Barbour RS. Checklists for improving rigour in qualitative research: a case of the tail wagging the dog? BMJ. 2001;322:1115-7.

93. Patton MQ. Enhancing the quality and credibility of qualitative analysis. Health Serv Res. 1999;34:1189-208.

94. Gradinger F, Britten N, Wyatt K, Froggatt K, Gibson A, Jacoby A, et al. Values associated with public involvement in health and social care research: a narrative review. Health Expect. 2015;18:661-75.

\section{Submit your next manuscript to BioMed Central and we will help you at every step:}

- We accept pre-submission inquiries

- Our selector tool helps you to find the most relevant journal

- We provide round the clock customer support

- Convenient online submission

- Thorough peer review

- Inclusion in PubMed and all major indexing services

- Maximum visibility for your research

Submit your manuscript at www.biomedcentral.com/submit 